Revista Brasil. Bot., V.31, n.4, p.667-678, out.-dez. 2008

\title{
Fungos anamorfos do solo da região dos lagos no Município de Santa Gertrudes, SP, Brasil ${ }^{1}$
}

\author{
NORBERTO CARLOS SCHOENLEIN ${ }^{2,5}$, CARLOS RENATO CORSO ${ }^{3}$, \\ IRACEMA HELENA SCHOENLEIN-CRUSIUS ${ }^{4}$, JOSÉ IVANILDO DE SOUZA ${ }^{4}$ e \\ LUÍSA HELENA DOS SANTOS OLIVEIRA ${ }^{4}$
}

(recebido: 15 de julho de 2007; aceito: 18 de setembro de 2008)

\begin{abstract}
Anamorphic fungi in the soil of the lake region in the municipal district of Santa Gertrudes, SP, Brazil). The soil of the ceramic pole in the Santa Gertrudes town, São Paulo State, Brazil, has been polluted since decades with several chemical elements, mainly lead and zinc. Four soil samples were collected, two during rainy and two during dry seasons, at five sites during November 2002 to June 2003, and measures of temperature, pH, lead, zinc and moisture soil contents were made. Fungi were obtained by Warcup's soil-plate method, modified by application of $1 \mathrm{~cm}^{3}$ of aqueous soil suspensions (1:10) over malt agar (2\%), added with $\mathrm{Zn}\left(\mathrm{NO}_{3}\right)_{2}$ or $\mathrm{Pb}\left(\mathrm{NO}_{3}\right)_{2}$ in increasing concentrations: $0,100,200,500$ or $1,000 \mathrm{mg} \mathrm{dm}^{-3}$. After five days of incubation at $25^{\circ} \mathrm{C}$, the colonies were purified and identified. Seventy anamorphic fungi were obtained, with $70 \%$ of similarity between mycotas isolated from the media added with metals. Forty-three taxa were isolated from culture media with $\mathrm{Pb}\left(\mathrm{NO}_{3}\right)_{2}$, with predominance of isolations in higher concentrations $(500$ and $1,000 \mathrm{mg}$ $\left.\mathrm{dm}^{-3}\right)$. Sixty-three taxa were obtained in the media with $\mathrm{Zn}\left(\mathrm{NO}_{3}\right)_{2}$, mainly in moderate and high concentrations (200 and $500 \mathrm{mg} \mathrm{dm}^{-3}$ ). Trichoderma spp., Penicillium spp. and several fungi species usually found in decomposing plant debris prevailed. The tendency to obtain higher numbers of fungal taxa in culture media with moderate to high concentrations of $\mathrm{Zn}$ and $\mathrm{Pb}$ may be justified by the existence of well adapted and competitive soil mycota, characterized by a high tolerance to metals and an efficient saprobic competitive ability.
\end{abstract}

Key words - anamorphs, ceramic pole, environmental impact, fungal diversity, heavy metals

RESUMO - (Fungos anamorfos do solo da região dos lagos no Município de Santa Gertrudes, SP, Brasil). O solo do pólo cerâmico no município de Santa Gertrudes, SP, tem sido poluído há décadas por diversos elementos químicos, principalmente chumbo e zinco. Foram realizadas quatro coletas de amostras de solo, duas durante a época chuvosa e duas na seca, em cinco locais, de novembro de 2002 a junho de 2003, determinando-se temperatura, pH, teores de chumbo e zinco e a umidade do solo. Os fungos foram isolados pelo método de Warcup, modificado pelo preparo de suspensões aquosas de solo (1:10) e aplicação de $1 \mathrm{~cm}^{3}$ das suspensões sobre malte agar (2\%), adicionado de $\mathrm{Zn}\left(\mathrm{NO}_{3}\right)_{2}$ e $\mathrm{Pb}\left(\mathrm{NO}_{3}\right)_{2}$ em concentrações crescentes: $0,100,200,500$ e $1.000 \mathrm{mg} \mathrm{dm}^{-3}$. Após cinco dias de incubação a $25^{\circ} \mathrm{C}$, as colônias foram purificadas e identificadas. Foram obtidos 70 táxons de fungos anamorfos, com $70 \%$ de similaridade entre as micotas obtidas nos meios com os dois metais. Foram isolados 43 táxons nos meios de cultura com $\mathrm{Pb}\left(\mathrm{NO}_{3}\right)_{2}$, com predominância deles nas concentrações mais elevadas (500 a $1.000 \mathrm{mg} \mathrm{dm}^{-3}$ ). Foram obtidos 63 táxons nos meios com $\mathrm{Zn}\left(\mathrm{NO}_{3}\right)_{2}$, principalmente nas concentrações moderada e elevada (200 e $500 \mathrm{mg} \mathrm{dm}^{-3}$ ). Prevaleceram espécies de Trichoderma, de Penicillium e diversos fungos que são encontrados associados a substratos vegetais em decomposição. A tendência de se obter número elevado de táxons em meios de cultura com concentrações moderadas a elevadas de $\mathrm{Zn}$ e $\mathrm{Pb}$ pode ser justificada pela existência de bem adaptada e competitiva micota do solo, caracterizada por elevada capacidade de tolerância aos metais e eficiente habilidade sapróbia competitiva.

Palavras-chave - diversidade fúngica, fungos anamorfos, impacto ambiental, metais pesados, pólo cerâmico

1. Parte da tese de doutorado do primeiro autor, curso de pós-graduação em Ciências Biológicas, área de Microbiologia Aplicada, UNESP, Rio Claro.

2. Universidade Guarulhos, Centro de Pós-Graduação, Pesquisa e Extensão, Praça Tereza Cristina noำ 1, 13067-067 Guarulhos, SP, Brasil.

3. Universidade Estadual Paulista, Departamento de Bioquímica e Microbiologia, Av. 24 A no 1515, Bela Vista, 13506-900 Rio Claro, SP, Brasil.

4. Instituto de Botânica, Seção de Micologia e Liquenologia, Caixa Postal 3005, 01061-970 São Paulo, SP, Brasil.

5. Autor para correspondência: norbertocs@ terra.com.br

\section{Introdução}

Os efeitos tóxicos dos metais pesados sobre os fungos despertaram interesse científico devido ao uso de elementos como cobre, chumbo e zinco na formulação de diversos defensivos agrícolas (Ross 1975). A elevação da quantidade e da variabilidade de poluentes à base de metais que vem sendo gradativamente depositados no ambiente norteou os estudos para aspectos ecológicos, relacionados com o diagnóstico da situação dos sistemas 
impactados, além de medidas de recuperação e conservação (Wainwright \& Gadd 1997).

A habilidade dos fungos sobreviverem à presença de metais tóxicos depende de diversas propriedades bioquímicas e estruturais, além de adaptações fisiológicas, morfológicas, genéticas e modificações do próprio metal com relação à especiação, disponibilidade e toxidez (Gadd 1992). Podem ser citadas também, interações como a precipitação ou complexação dos metais pesados por produtos metabólicos excretados pelos fungos, a fixação dos metais à parede celular, a composição da parede, o tipo e a espécie metálica envolvida, o transporte de metais tóxicos através da membrana plasmática, a fixação intracelular dos metais às proteínas e peptídios e a transformação de uma espécie metálica nociva em uma forma menos danosa (Gadd 1993, Kushner 1993, Ross 1993).

Estudos de Tatsuyama et al. (1974, 1975, 1977) no Japão, revelaram que os solos poluídos por elevadas doses de metais apresentavam diversidade de fungos reduzida, mas com grande ocorrência de espécies cosmopolitas e tolerantes como as de Fusarium, Penicillium e Trichoderma. Considera-se que a presença de determinados elementos metálicos pode causar redução da abundância de algumas espécies ou comprometer a sua diversidade, como verificado por Nordgren et al. $(1983,1985)$ em solo de região de mineração em Gusum, no sul da Suécia. Além disso, um elemento químico tóxico, quando localizado no interior de células fúngicas em concentrações sub-letais, pode sofrer alterações físico-químicas e tornar-se mais ou menos tóxico, ser imobilizado na biomassa ou ser translocado para regiões específicas das células, sendo transferido à cadeia trófica por ingestão, extravazamento ou morte do micélio (Dighton 2003).

O efeito seletivo dos metais pode resultar na substituição de diversas espécies e no estabelecimento de uma micota terrestre resistente ou tolerante (Jordan $\&$ Lechevalier 1975). A presença de determinado metal no solo representa, assim, mais um parâmetro a ser levado em consideração na compreensão das interações competitivas que contribuem para a composição da micota terrestre. Havendo comprometimento da diversidade de fungos que promovem a decomposição da matéria orgânica, os efeitos danosos de metais pesados podem ser potencializados (Hendricks 1996).

Existe interesse no conhecimento da diversidade dos fungos do solo, principalmente dos ecossistemas tropicais, para se avaliar o perfil microbiológico do ambiente e a potencialidade de recuperação em casos de impactos. No Brasil, com relação aos efeitos de metais pesados sobre a diversidade de fungos, em levantamento de fungos zoospóricos, anamorfos, micorrízicos e zigomicetos na região do vale do Rio Mogi e Vale do Rio Pilões em Cubatão, SP, foi observado que a redução do número de táxons da micota depende, em grande parte, do grupo taxonômico ao qual os fungos pertencem. Fungos anamorfos, por exemplo, são representados por espécies com maior adaptabilidade às condições ambientais do que espécies de Mucorales (Schoenlein-Crusius et al. 2006). Recentemente foi realizado levantamento de Mucorales no solo de mineração de cobre e a avaliação da produção de amilase e inulinase por algumas espécies, verificando-se efeito negativo do impacto ambiental na abundância das espécies de fungos nos solos da região de Jaguarari, Bahia (Santiago \& Souza-Motta 2006). Na região da Represa do Guarapiranga, em São Paulo, a micota do solo, composta predominantemente por fungos anamorfos cosmopolitas, apresentou-se mais afetada pela mudança do perfil da vegetação do que pela presença de cobre (Santos et al. 1998).

A região escolhida para o presente estudo, conhecida como "Região dos Lagos de Santa Gertrudes", situada no pólo cerâmico desse município, está inserida na bacia hidrográfica do córrego da Fazenda Itaqui; este é um afluente do rio Corumbataí e atravessa a região estudada. Os lagos não são naturais, mas resultantes de antigas cavas de extração de argila da Formação Corumbataí.

O pólo cerâmico de Santa Gertrudes é o maior do gênero no país e responsável por mais da metade da produção nacional de pisos e revestimentos cerâmicos esmaltados. Nessa área, foram dispostos inadequadamente resíduos da esmaltação de pisos e revestimentos (óxidos metálicos e outras formas de elementos químicos) gerando contaminação ambiental; além disso, houve o avanço de aterros por parte das indústrias, causando intensas modificações na paisagem (Bonacin-Silva 2001).

Ao longo das últimas duas décadas, a ação conjunta de diversos órgãos governamentais e da iniciativa privada criaram o "Projeto Corumbataí Cerâmicas", no qual diversas ações foram tomadas para diagnosticar e subsidiar medidas de recuperação da área, sendo que diversos parâmetros químicos, físico-químicos e geológicos foram analisados, avaliando-se as áreas críticas e passíveis de monitoramento (Cetesb 2001). Não existem, porém, dados publicados sobre os microrganismos, os quais são reconhecidamente responsáveis pela manutenção da fertilidade do solo (Primavesi 1990). O presente estudo teve por objetivo contribuir para a ampliação dos conhecimentos sobre a região, fornecendo informações sobre a ocorrência de 
fungos anamorfos no solo, bem como o comportamento dos táxons isolados em meio de cultura com diferentes concentrações de chumbo e zinco.

\section{Material e métodos}

Local de coleta - Na altura do quilômetro 168 da Rodovia Washington Luís (SP-310), à margem esquerda no sentido capital-interior, foram escolhidos quatro locais para amostragens de solo na região do pólo cerâmico de Santa Gertrudes. Estes foram caracterizados por Bonacin-Silva (2001) como severamente afetados por chumbo (15 a 14.700 mg kg-1) e zinco (53 a $2.930 \mathrm{mg} \mathrm{kg}^{-1}$ ), além de um local distante destes, inicialmente considerado controle, localizado fora do pólo cerâmico (designado por local 5 neste trabalho). Amostras de solo (cinco amostras compostas por local e coleta) foram coletadas até $10 \mathrm{~cm}$ de profundidade em quatro visitas ao campo, de novembro de 2002 a junho de 2003. Foi efetuada análise dos teores de chumbo e zinco $\left(\mathrm{mg} \mathrm{kg}^{-1}\right)$ das amostras do solo, através do método do plasma de indução acoplada (ICP), no Instituto Campineiro de Análise de Solo e Adubos S/C Ltda.

As coordenadas geográficas dos pontos de amostragem foram determinadas com G.P.S. (Global Positioning System) Garmin. Os locais de coleta foram assim caracterizados: local 1 - área com vegetação rala e de solo seco, localizada a $22^{\circ} 27^{\prime} 46^{\prime \prime} \mathrm{S}$ e $47^{\circ} 32^{\prime} 29^{\prime \prime} \mathrm{W}$, com o solo contendo de 2,44 a 4,48 $\mathrm{mg}^{-1}$ de zinco e 0,68 a $1,0 \mathrm{mg} \mathrm{kg}^{-1}$ de chumbo; local 2 - área coberta com pastagens e solo pantanoso, localizada a $22^{\circ} 27^{\prime} 49^{\prime \prime} \mathrm{S}$ e $47^{\circ} 32$ ' $32^{\prime \prime} \mathrm{W}$, solo com 0,82 a $22,80 \mathrm{mg} \mathrm{kg}^{-1}$ de zinco e 1,19 a 45,16 mg kg-1 de chumbo; local 3 - área situada na região central do pólo cerâmico, situada a $22^{\circ} 27^{\prime} 58^{\prime \prime} \mathrm{S}$ e $47^{\circ} 32$ ' $22^{\prime \prime} \mathrm{W}$, solo com 3,80 a $34,20 \mathrm{mg} \mathrm{kg}^{-1}$ de zinco e 7,42 a 908,40 $\mathrm{mg} \mathrm{kg}^{-1}$ de chumbo; local 4 - região pantanosa entre dois lagos pesqueiros, localizada a $22^{\circ} 27^{\prime} 59^{\prime \prime} \mathrm{S}$ e 47 32' $22^{\prime}$ ' W, solo com 10,04 a $35,40 \mathrm{mg} \mathrm{kg}^{-1}$ de zinco e 58,00 a $340,40 \mathrm{mg} \mathrm{kg}^{-1}$ de chumbo e local 5 - localizado em um jardim público no perímetro urbano de Santa Gertrudes, a $22^{\circ} 27^{\prime} 12^{\prime \prime} \mathrm{S}$ e $47^{\circ} 32^{\prime} 39^{\prime \prime} \mathrm{W}$, com solo contendo de 5,14 a $6,74 \mathrm{mg} \mathrm{kg}^{-1}$ de zinco e de 3,48 a $5,79 \mathrm{mg} \mathrm{kg}^{-1}$ de chumbo. De acordo com os critérios da Cetesb (2001) para a classificação de áreas quanto ao grau de poluição por metais pesados, somente os locais 3 e 4 apresentam solos cujos valores superaram os índices máximos que justificam uma intervenção, isto é, 900 e $300 \mathrm{mg} \mathrm{kg}^{-1}$ de chumbo, respectivamente para regiões industriais e residenciais.

Concomitantemente às coletas de solo, foram medidas as temperaturas a $20 \mathrm{~cm}$ de profundidade, com termômetro de solo. $\mathrm{O}$ teor de umidade das amostras de solo foi medido por meio da determinação do peso seco de $3 \mathrm{~g}$ de solo úmido, após 20 minutos de exposição a $105^{\circ} \mathrm{C}$, em balança Marte ID50. O pH foi determinado preparando-se uma suspensão de $40 \mathrm{~g}$ de solo em $100 \mathrm{~cm}^{3}$ de água destilada (Gazarini 1983). Dados da precipitação pluviométrica em milímetros nos dias das coletas foram fornecidos pela estação meteorológica do Ceapla (Centro de Análise e Planejamento Ambiental, UNESP, Rio Claro, SP).

Isolamento e identificação dos fungos - $\mathrm{O}$ isolamento dos fungos foi feito pelo método da placa de solo Warcup (1950), modificado pelo preparo de suspensões de solo $\left(10^{-1}\right)$, devido ao elevado estado de coesão do solo argiloso. Dez gramas de solo das amostras compostas referentes a cada um dos cinco locais foram diluídos com $100 \mathrm{~cm}^{3}$ de água destilada esterilizada, em erlenmeyers de $250 \mathrm{~cm}^{3}$. Os frascos foram fechados com filme de PVC, mantidos sob agitação orbital (a $120 \mathrm{rpm}$ ) por 90 minutos, retirados da mesa agitadora e deixados em repouso por 30 minutos, sendo que $1 \mathrm{~cm}^{3}$ das suspensões foi espalhado sobre placas de Petri contendo malte agar (2\%), adicionado de concentrações crescentes de $\mathrm{Pb}\left(\mathrm{NO}_{3}\right)_{2}$ ou $\mathrm{Zn}\left(\mathrm{NO}_{3}\right)_{2}$, isto é, 0 (SM), 100, 200, 500 e $1.000 \mathrm{mg} \mathrm{dm}^{-3}$, em triplicata. As placas foram incubadas durante sete dias em ambiente climatizado, entre 20 a $23^{\circ} \mathrm{C}$. As colônias foram transferidas para meio BDA (batata $140 \mathrm{~g}$, dextrose $10 \mathrm{~g}$, agar $20 \mathrm{~g}$ e $1 \mathrm{dm}^{3}$ de água destilada) e novamente incubadas durante sete dias. Os fungos foram identificados através da observação de estruturas micro e macroscópicas e consultas às chaves de identificação pertinentes e posteriormente preservados pelo método de Castellani (Schoenlein-Crusius et al. 1994).

Para comparar as micotas obtidas entre as concentrações de metais no meio de cultivo, locais de coleta e épocas chuvosa e seca, foi utilizado o índice de similaridade de Sörensen, calculado segundo a fórmula:

$$
\mathrm{S}=\left[2 \mathrm{~W} \times(\mathrm{A}+\mathrm{B})^{-1}\right] \times 100
$$

Onde: $\mathrm{A}=$ somatória das ocorrências de todos os fungos isolados na situação a; $\mathrm{B}=$ somatória das ocorrências de todos os fungos isolados na situação b e W = somatória das ocorrências dos fungos isolados comuns às duas situações.

\section{Resultados e discussão}

Caracterização climática das áreas de estudo - De acordo com os dados meteorológicos fornecidos pelo Ceapla, as coletas de 15 de novembro de 2002 e 18 de março de 2003 foram realizadas em época chuvosa, sendo que nestes dias as precipitações pluviométricas corresponderam a 27,6 e 27,1 mm, respectivamente, com acúmulo de $288,2 \mathrm{~mm}$ em novembro de 2002 e 96,9 mm em março de 2003. Em 28 de maio de 2003 e 30 de junho de 2003 não ocorreram chuvas, havendo o acúmulo de 45,8 mm em maio e 15,6 mm em junho, situando estes meses em época climática seca. Estes dados meteorológicos confirmam que o clima de Santa Gertrudes é do tipo Cwa (Sistema Köppen), subtropical, seco no inverno e chuvoso no verão.

Com relação às variáveis abióticas medidas em campo, a temperatura do solo variou de $21^{\circ} \mathrm{C}$ na $1^{\text {a }}$ coleta, $24^{\circ} \mathrm{C}$ na $2^{\text {a }}, 25^{\circ} \mathrm{C}$ na $3^{\mathrm{a}}$ e $24^{\circ} \mathrm{C}$ na $4^{\text {a }}$ coleta. Os 
valores médios da umidade do solo variaram em torno de $17 \%$ na $1^{\text {a }}$ coleta, $15 \%$ na $2^{\text {a }}$ e $3^{\text {a }}$ coletas e $7 \%$ na $4^{\text {a }}$ coleta. Com relação ao $\mathrm{pH}$, os valores apresentaram poucas variações, tanto entre os locais quanto entre as coletas, situando-se entre 5,40 a 6,80 , representando valores maiores do que os esperados para a região, que correspondem de 3,5 a 4,0 (Bonacin-Silva 2001). Com exceção do $\mathrm{pH}$, as características do solo condizem com os resultados obtidos em levantamento pedológico e geológico da região (Bonacin-Silva 2001). Sob o ponto de vista micológico, pode-se considerar que as variações abióticas situam-se dentro da faixa tolerada pelos fungos (Domsch et al. 1980).

Diversidade dos fungos - O número de táxons obtidos, isto é 70, é maior do que o mencionado em estudos nos quais foram usadas metodologias similares. Assim, das áreas afetadas por poluição em Cubatão, SP, foram obtidos 43 táxons de fungos anamorfos (SchoenleinCrusius et al. 2006); em levantamentos dos fungos terrestres às margens da Represa do Guarapiranga, na cidade de São Paulo, foram obtidos 60 táxons em áreas muito afetadas por influência antrópica e poluição por sulfato de cobre (Santos et al. 1998), afetando também 41 táxons de fungos filamentosos, além de decompositores de folhas dessa região (Wellbaum et al. 1999).

Os meios de cultura sem adição de chumbo ou de zinco foram usados como controles, podendo considerar-se surpreendente a baixa ocorrência de fungos isolados nestas condições. Os táxons Trichoderma aureoviride Rifai, T. pseudokoningii Rifai, T. viride Pers. ex Gray e Cladosporium herbarum (Pers.) Link ex. S. F. Gray foram predominantes nas amostras procedentes das $3^{\underline{a}} \mathrm{e}$ 4ㄹ coletas conduzidas na época seca.

O índice de similaridade de Sörensen, comparando a micota obtida nos meios de cultura acrescidos de chumbo com a obtida nos meios acrescidos de zinco, correspondeu a 70\%. Christensen (1989) considera como elevado o índice acima de $60 \%$, para ecossistemas nos quais a biodiversidade é grande, como geralmente ocorre nos trópicos.

Foram obtidos 43 táxons no meio de cultura adicionado de $\mathrm{Pb}\left(\mathrm{NO}_{3}\right)_{2}$ (tabela 1). As maiores ocorrências foram de Trichoderma viride Pers. ex Gray (27 ocorrências), T. pseudokoningii Rifai (18 registros), Penicillium variabile Soop (13 registros), T. aureoviride Rifai (12 registros), $P$. purpurogenum Stoll (9 registros) e $P$. janthinellum Biourge (9 registros). Na primeira coleta foram obtidos 14 táxons, na segunda 22, na terceira 21 e na última 15. O número total de táxons obtidos nas duas coletas realizadas na época chuvosa e nas duas realizadas na época seca foi equivalente, correspondendo a 29. Na época chuvosa, porém, foram obtidos 15 táxons, 11 na época seca e 17 foram registrados nas duas épocas climáticas. O índice de similaridade de Sörensen entre as duas micotas correspondeu a $58 \%$. De acordo com Christensen (1989), este índice pode ser considerado baixo, indicando a existência de diferenças qualitativas na composição das micotas, apesar das semelhanças quantitativas no número de táxons.

Com relação à influência das doses crescentes de $\mathrm{Pb}\left(\mathrm{NO}_{3}\right)_{2}$ nos meios de cultura em todas as coletas, verificou-se a tendência de ocorrer número elevado de táxons nos meios de cultura com concentrações mais altas de chumbo, isto é, entre 500 e $1.000 \mathrm{mg} \mathrm{dm}^{-3}$ (tabela 1). Número elevado de táxons, correspondentes a 17 , 10 e 9 foram registrados nos meios de cultura adicionados de $1000 \mathrm{mg} \mathrm{dm}^{-3}$, além dos 14 táxons isolados em meio de cultura acrescido de $500 \mathrm{mg} \mathrm{dm}^{-3}$.

A observação de que algumas espécies tais como Cladosporium cladosporioides (Fresen.) de Vries, Nigrospora sphaerica (Sacc.) Mason, Penicillium citreonigrum Dierckx, $P$. griseoroseum Dierckx e $P$. waksmanii K. M. Zalessky foram isolados somente nos meios de cultura com acréscimo de concentrações máximas de chumbo reforçam a tendência destes fungos, que geralmente são isolados de quaisquer outros substratos e meios de cultura, tolerarem a presença de altas concentrações de metais pesados (Domsch et al. 1980).

O número de táxons registrados nos locais de coleta, em ordem decrescente dos teores de metais presentes no solo foi: 11 táxons (local 3), 14 táxons (local 4), 26 táxons (local 2), 14 (local 5) e 23 (local 1). Os índices de similaridade entre as micotas obtidas nos cinco locais de coleta foram baixos, entre $45 \%$ e $50 \%$, havendo diversos táxons exclusivos de apenas um local de coleta.

Foram obtidos 63 táxons no meio de cultura adicionado de $\mathrm{Zn}\left(\mathrm{NO}_{3}\right)_{2}$ (tabela 2). As maiores ocorrências foram para Trichoderma viride Pers. ex Gray (18 ocorrências), Paecilomyces lilacinus (Thom) Samson (17), Penicillium restrictum J. C. Gilman \& E. V. Abbott (16), Fusarium oxysporum Schlecht (12) e P. purpurogenum Stoll (10). Na primeira coleta foram obtidos 16 táxons, na segunda 25 , na terceira 31 e na última 43 . O número total de táxons obtidos correspondeu a 34 nas duas coletas realizadas na época chuvosa e a 54 nas coletas realizadas na época seca. Vinte e sete táxons ocorreram na época seca e 15 taxons foram obtidos exclusivamente na época chuvosa, sendo que tanto na época de seca como na de chuva ocorreram 28 táxons. $O$ índice de similaridade de Sörensen entre as micotas isoladas em épocas climáticamente distintas correspondeu a 54\%, indicando baixa semelhança na composição das mesmas. 
Revista Brasil. Bot., V.31, n.4, p.667-678, out.-dez. 2008

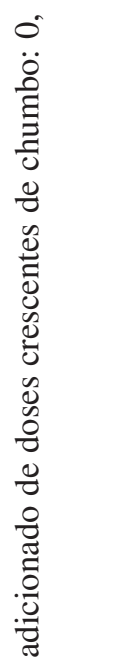

究

苋草

善

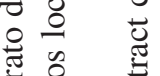

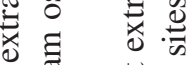

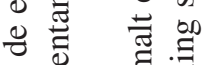

잉

递 递

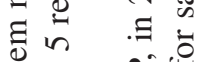

$\hat{n}$ की

is

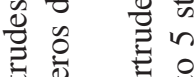

苞苛

苞藏

㱐

次

음 吾

등

吾焉

玡

궁

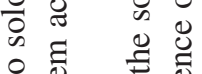

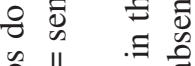

总就表

ज क ज्ञ

of है

总驾

드의

흥휴

ह छ च

玨

-

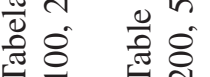

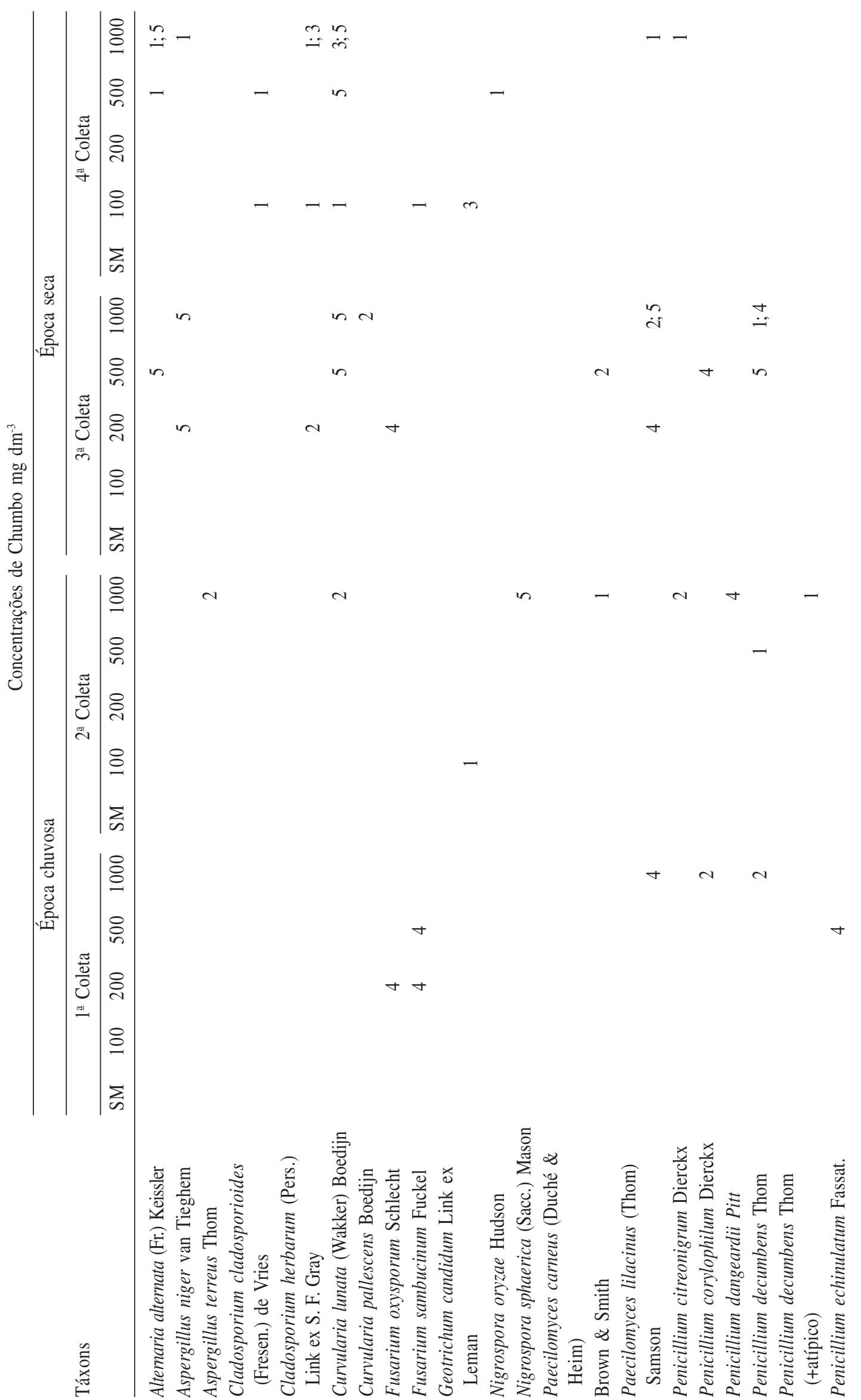




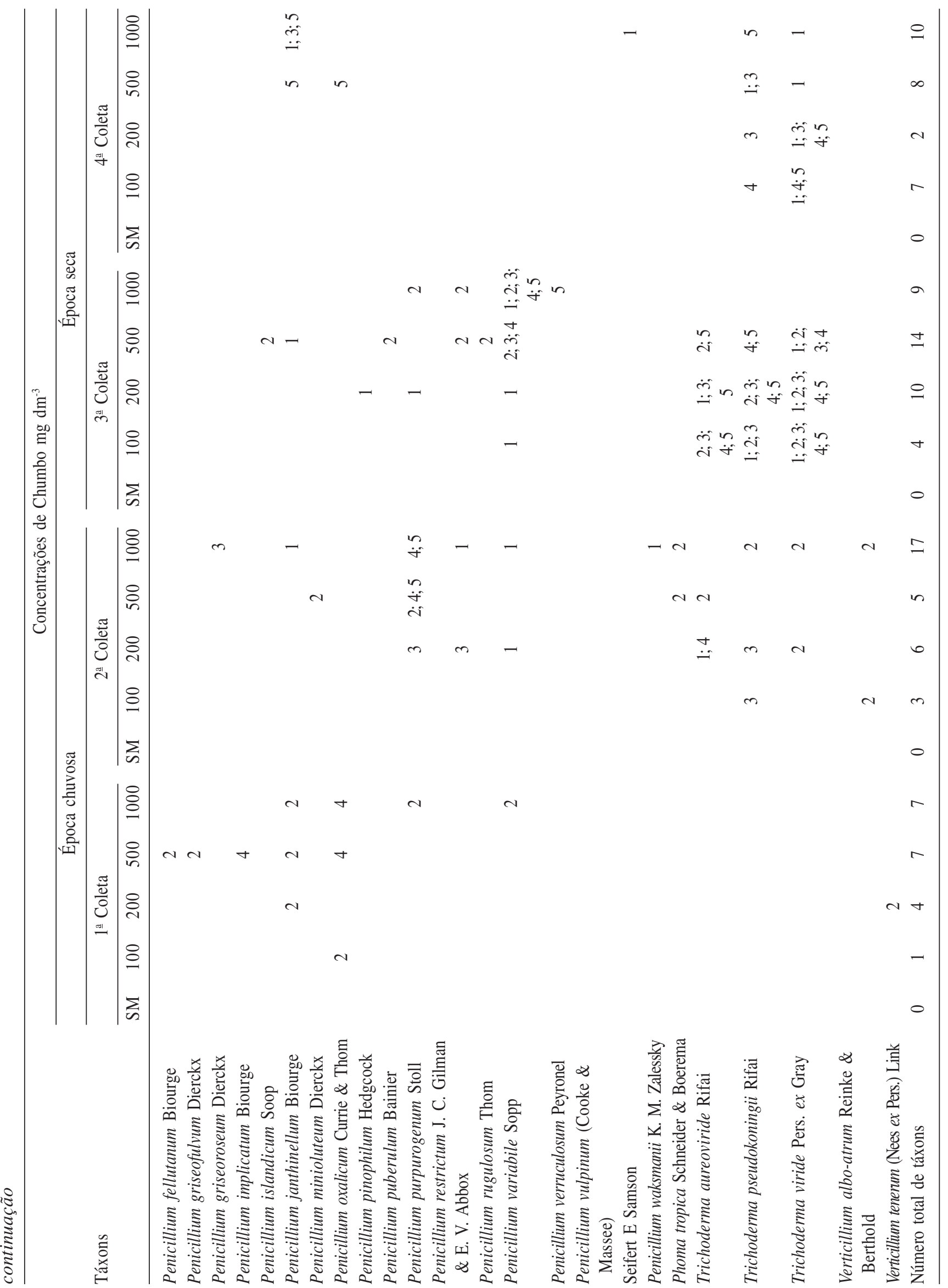


Revista Brasil. Bot., V.31, n.4, p.667-678, out.-dez. 2008

673

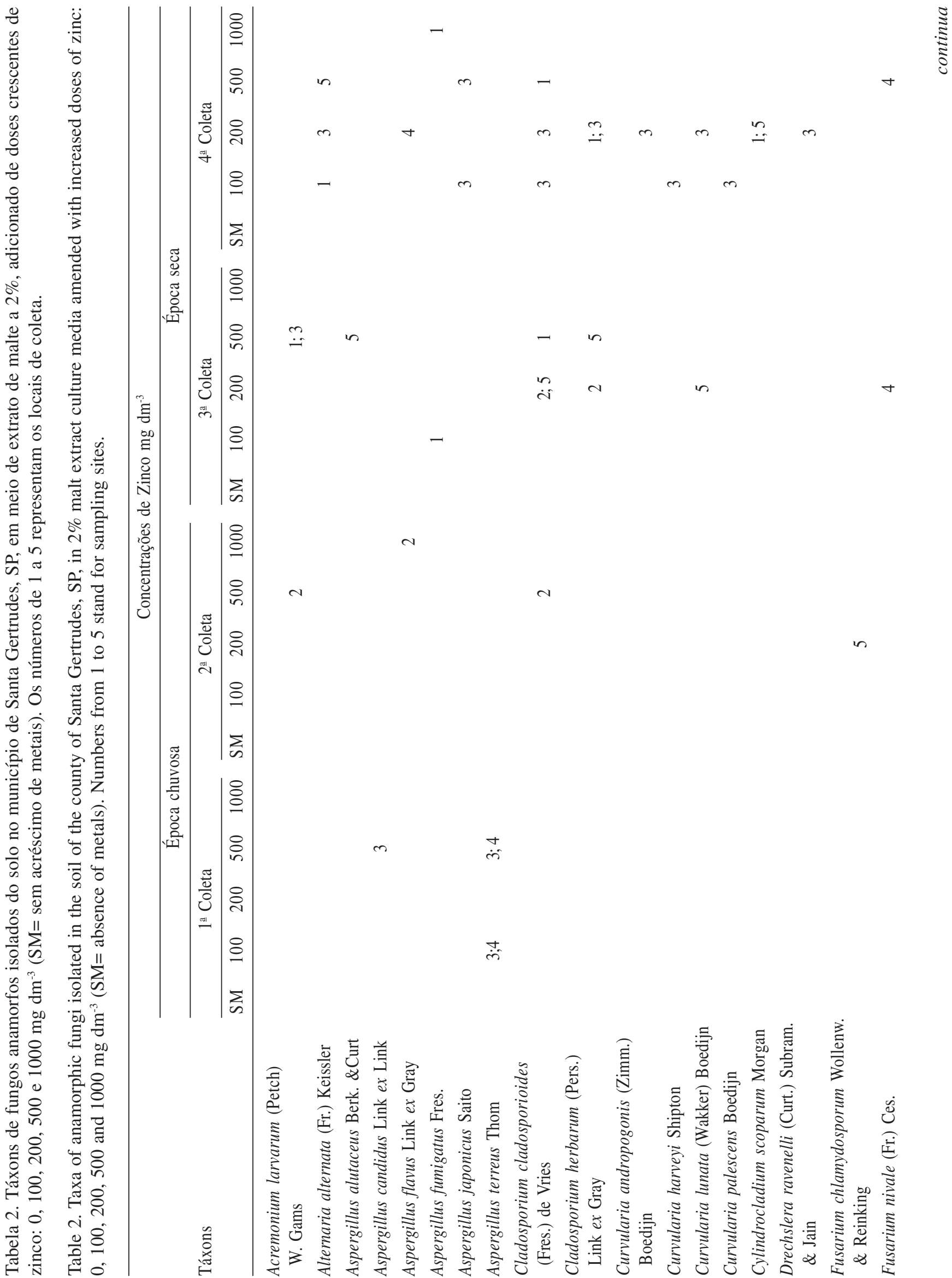


N. C. Schoenlein et al.: Fungos anamorfos no Município de Santa Gertrudes, SP

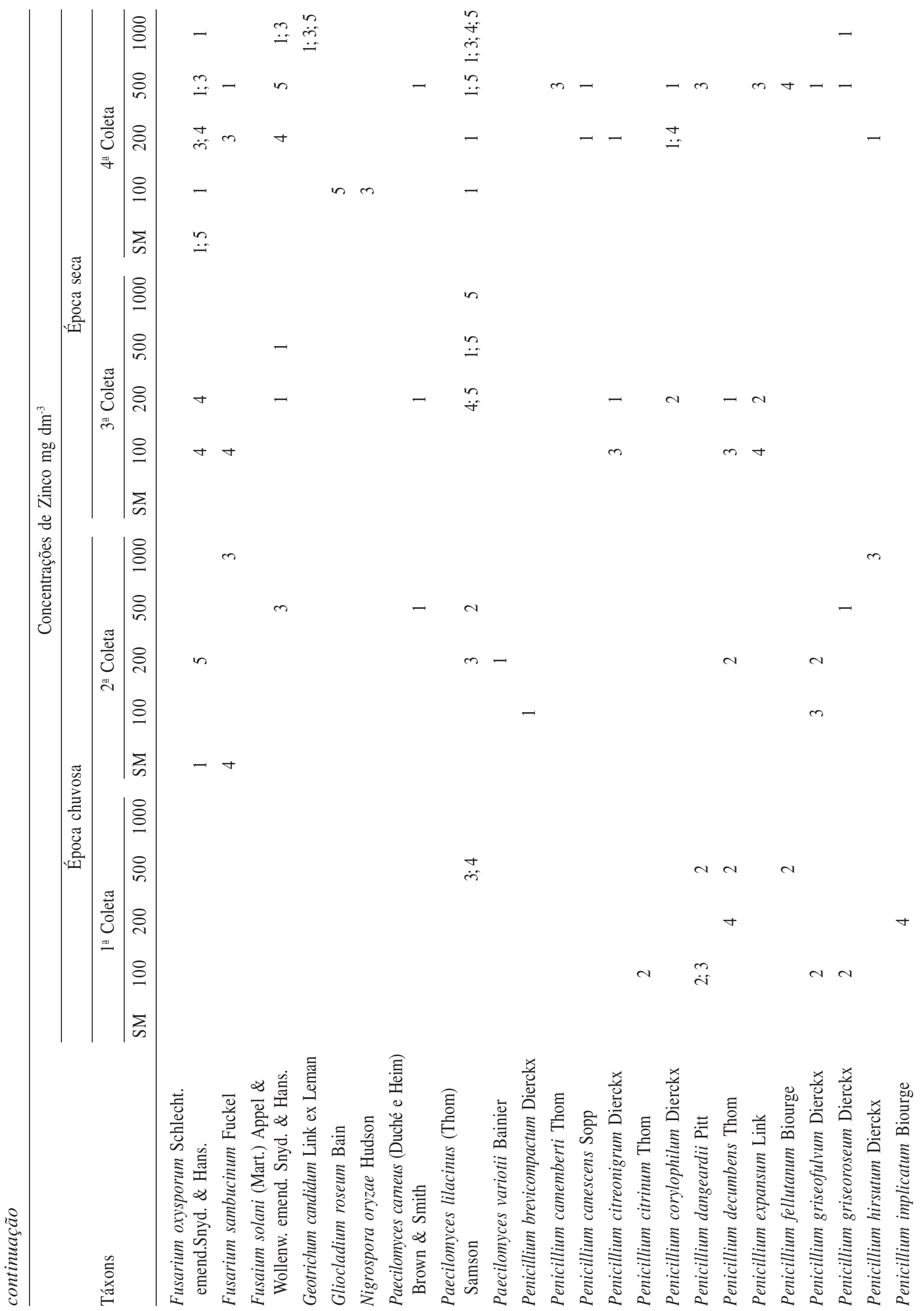


Revista Brasil. Bot., V.31, n.4, p.667-678, out.-dez. 2008

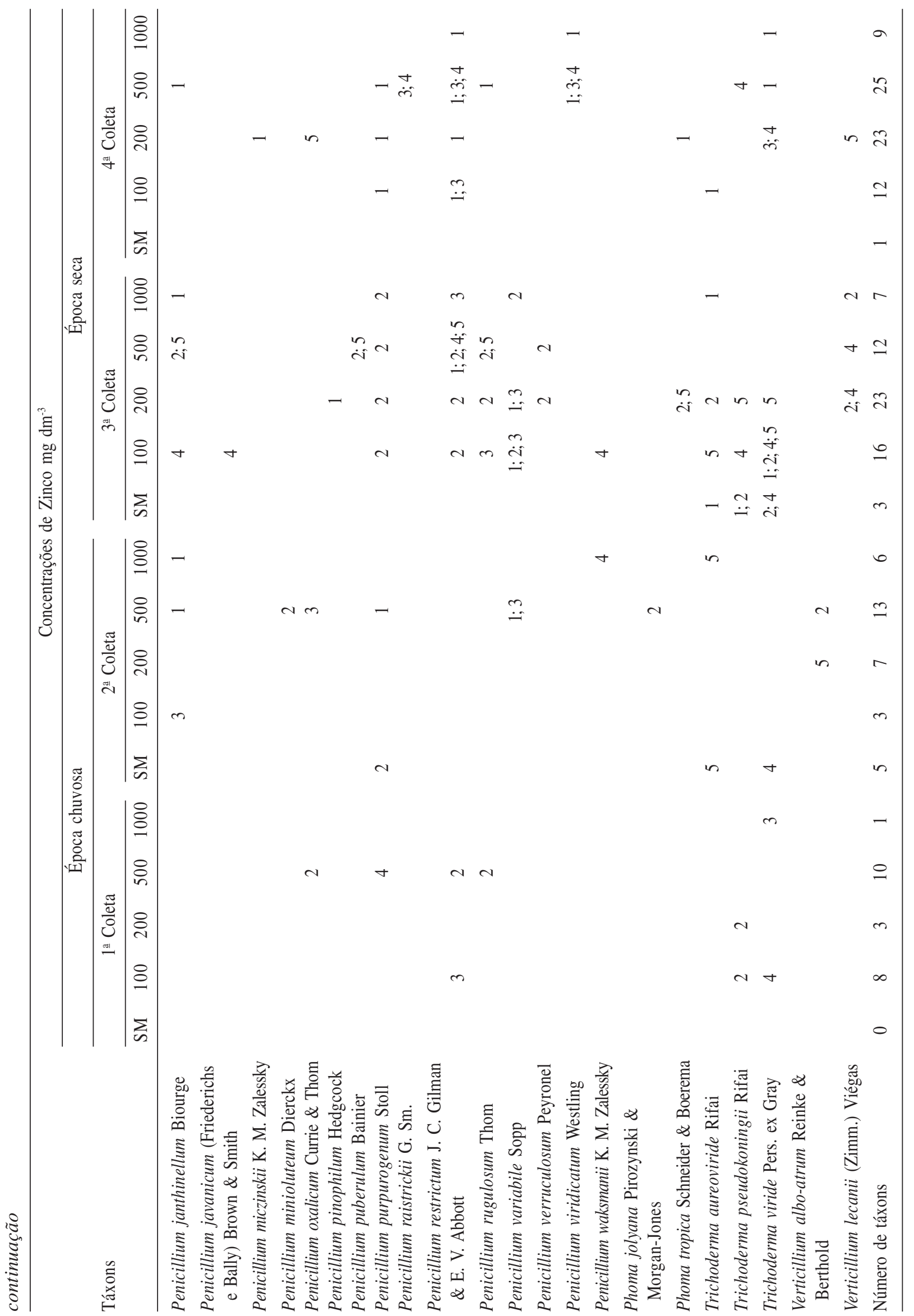


Com relação à influência das doses crescentes de $\mathrm{Zn}\left(\mathrm{NO}_{3}\right)_{2}$ no meio de cultura, verificou-se elevado número de táxons (23 e 25) nas concentrações de 200 e $500 \mathrm{mg} \mathrm{dm}^{-3}$ respectivamente. Observou-se, também, maior número de fungos nos meios contendo zinco do que nos adicionados com chumbo. Somente Geotrichum candidum Link ex Lehman foi isolado exclusivamente em meio com concentração máxima de chumbo $\left(1000 \mathrm{mg} \mathrm{dm}^{-3}\right)$.

O número de táxons registrados nos locais de coleta, em ordem decrescente das concentrações de zinco no solo foi: 33 táxons (local 3), 22 táxons (local 4), 29 táxons (local 2), 23 (local 5) e 34 (local 1). Os índices de similaridade entre as micotas obtidas nos cinco locais de coleta foram baixos, entre $45 \%$ e $57 \%$, havendo diversos táxons que ocorreram somente em determinado local de coleta. Nove espécies ocorreram exclusivamente no local 3 , quatro ocorreram somente no local 4 , cinco no local 2 , três foram registrados somente no local 5 e oito no local 1.

Os resultados indicaram que a diversidade dos fungos provavelmente é menos afetada pela presença de zinco do que de chumbo. Nas concentrações inferiores a $100 \mu \mathrm{M}$, o zinco é considerado elemento essencial para o crescimento de fungos (Ross 1975). A partir desta dosagem, o zinco geralmente causa efeitos prejudiciais acentuados sobre a vegetação e a microbiota (Jordan \& Lechevalier 1975).

Os táxons obtidos no presente estudo fazem parte da micota cosmopolita, presente em praticamente todos os ambientes terrestres. Os gêneros Aspergillus, Fusarium, Penicillium e Trichoderma são considerados comuns no solo (Domsch et al. 1980). No ambiente estudado, verificou-se que há poucas espécies raras ou diferentes de qualquer levantamento de fungos do solo (SchoenleinCrusius et al. 2006) e portanto pode ser altamente resistente às diferentes condições ambientais, tornandose predominante ao longo do tempo em que o solo foi submetido ao impacto causado pela presença dos metais na sua composição.

A ocorrência das espécies de Alternaria, Cladosporium, Curvularia, Nigrospora e Phoma, comumente associada a substratos vegetais em decomposição, ao lado de espécies de Trichoderma, com reconhecida habilidade para decompor celulose e hemicelulose, indica que a micota ainda conta com importantes decompositores da matéria orgânica (Paul \& Clark 1996).

A presença destes fungos não está associada exclusivamente a ambientes ricos em metais (Domsch et al. 1980); a obtenção dos mesmos em meios adicionados de elevadas concentrações de chumbo pode estar associada à elevação da tolerância ao metal ao longo do tempo ou a eliminação de eventuais outros fungos competidores, como citado na comparação entre os efeitos tóxicos de metais sobre fungos e bactérias (Rajapaksha et al. 2004). Nesse estudo verificou-se que os fungos são mais resistentes aos metais do que as bactérias.

Efeitos restritivos do cobre e do zinco sobre a biomassa fúngica e taxa de respiração do solo foram verificados por Nordgren et al. (1983) próximo a uma região de mineração no sul da Suécia, através de mudanças acentuadas na composição das micotas, com acréscimo em abundância de algumas espécies de Paecilomyces e Geomyces. Os mesmos autores verificaram acentuados decréscimos na frequência de isolamento de algumas espécies típicas do solo, seguida pela substituição de algumas espécies por outras, raras na região, como principal resultado dos efeitos da presença dos metais no solo.

A análise dos resultados dos levantamentos em áreas poluídas deve levar em consideração as limitações e seletividade impostas pelas técnicas de isolamento, concentrações dos metais e nível de adaptabilidade dos microrganismos aos metais. Carter (1978 apud Nordgren et al. 1983) reportou, por exemplo, uma modificação significativa na estrutura da comunidade microbiana em locais contaminados com elevados níveis de níquel. Freedman \& Hutchinson (1980), estudando a contaminação de níquel e cobre, não verificaram efeito algum, variando somente o tempo do estudo.

Metais altamente tóxicos como o chumbo não são considerados essenciais aos microrganismos, sendo que a presença dos mesmos no solo ou adicionados a meios de cultura é mais conhecida como causa de inibição de crescimento e redução da diversidade microbiana do que o contrário (Ross 1975). Elevações no número de táxons no solo poluído por metais pesados, de maneira geral, têm sido associadas a complexas interações microbianas competitivas, ainda pouco explicadas. Além disso, os efeitos de determinados metais no solo podem ser cumulativos, alterando as características pedológicas imprevisivelmente ao longo do tempo (Freedman \& Hutchinson 1980). A adaptação microbiana constitui um mecanismo importante nas respostas à presença de contaminação e pode resultar na compensação de determinado efeito adverso pela elevação da sua atividade ao longo do tempo (Duxbury \& Bicknell 1983). A frequência de isolamento de microrganismos tolerantes a metais pesados no solo pode aumentar, acompanhando o aumento dos níveis de metais, acarretando em diminuição da diversidade de espécies e mudança na composição da micota que, por conseguinte, pode resultar em redução na atividade funcional do solo (Fomina $e t$ al. 2005). 
Os fungos vivem naturalmente sob condições oligotróficas ou de estresse nutricional devido aos baixos níveis de carbono disponíveis na maioria dos solos e demais ambientes, sendo que nestas condições são mais sensíveis aos poluentes do que quando crescem em meios ricos em nutrientes (Wainwright \& Gadd 1997, Gadd et al. 2001). $\mathrm{O}$ efeito tóxico de metais no crescimento fúngico varia entre as diferentes espécies, entre diferentes linhagens da mesma espécie e depende da forma química em que o metal está presente, de modo que a diminuição dos efeitos tóxicos pode estar correlacionada com o aumento da concentração de carbono disponível (Fomina et al. 2005).

Fomina et al. (2000) observaram que Trichoderma viride Pers., Gliocladium roseum Bainier, Geotrichum candidum Link e Humicola grisea Traaen, crescendo sobre blocos de $1 \mathrm{~cm}^{2}$ de Czapek-Dox agar, suplementado com diferentes teores de sacarose $\left(1-30 \mathrm{~g} \mathrm{dm}^{-3}\right)$ e adicionado de cádmio ou cobre $(0,25-5,00 \mathrm{mM})$ apresentaram quimiotropismo negativo em direção aos blocos de meio de cultivo contendo metais, constatando que o tropismo negativo foi menor quando houve maior concentração de sacarose.

Quando se estuda a diversidade a partir de alguns gramas de solo (Kirk et al. 2004), tiram-se conclusões sobre a comunidade microbiana que eventualmente está presente naquele local. Segundo Trevors (1998), contudo, a dificuldade está em afirmar se aquela heterogeneidade não é inerente a uma população pré existente ou uma população que se adaptou àquele local.

Franklin \& Mills (2003) usaram múltiplas escalas espaciais, com intervalos de amostragem variando de $2,5 \mathrm{~cm}$ a $11 \mathrm{~m}$, para estudar a heterogeneidade espacial das comunidades microbianas do solo. Em solo de campo de trigo na região leste da Virginia, Estados Unidos da América, eles relataram que comunidades microbianas podem ter diversos níveis de organização e que elas poderiam ser dependentes de diferentes propriedades do solo ou grupos de propriedades modificadas do solo.

Por mais cuidadosos, circunscritos ou abrangentes que sejam os levantamentos de microrganismos terrestres em locais contaminados por metais pesados, os resultados obtidos expressam apenas uma parte da história de determinado poluente no solo. A caracterização da micota terrestre nativa como bem diversificada, em solos altamente impactados, deve ser considerada como sendo somente um indicativo de que ainda há condições favoráveis para a recuperação e conservação da região estudada.

Agradecimentos - Ao Técnico Sr. Roberto José Pedro do Departamento de Bioquímica e Microbiologia da Universidade Estadual Paulista, Rio Claro, SP, pelo auxílio prestado nas coletas de solo na região estudada.

\section{Referências bibliográficas}

BONACIN-SILVA, A.L. 2001. Caracterização ambiental e estudo do comportamento do chumbo, zinco e boro em área degradada por indústrias cerâmicas - região dos lagos de Santa Gertrudes, SP. Dissertação de mestrado, Universidade de São Paulo, São Paulo.

CETESB 2001. Parecer técnico № 08/00/EQSS de 26.07.2000. Avaliação ambiental das lagoas situadas na região do pólo cerâmico de Santa Gertrudes. Piracicaba, São Paulo.

CHRISTENSEN, M. 1989. A view of fungal ecology. Mycologia 81:1-19.

DIGHTON, J. 2003. Fungal interactions with humans. In Fungi in ecosystems processes. Dighton, J. (Ed), Marcel Dekker Inc., New York, p. 305-390.

DOMSCH, K.H., GAMS, W. \& ANDERSON, T.H. 1980. Compendium of soil fungi. Academic Press, New York.

DUXBURY, T. \& BICKNELL, B. 1983. Metal-tolerant bacterial populations from natural and metal-polluted soils. Soil Biology and Biochemistry 15:243-250.

FOMINA, M., BURFORD, E.P. \& GADD, G.M. 2005. Toxic metals and fungal communities. In: The fungal community: its organization and role in the ecosystem (J. Dighton, J. F. White \& P. Oudemans, eds.), Taylor \& Francis Publishers, Boca Raton. p.733-758.

FOMINA, M., RITZ, K. \& GADD, G.M. 2000. Negative chemotropism to toxic metals. Microbiology Letters 193:207-211.

FRANKLIN, R.B. \& MILLS, A.L. 2003. Multi-scale variation in spatial heterogeneity for microbial community structure in an eastern Virginia agricultural field. FEMS Microbiology Ecology 44:335-346.

FREEDMAN, B. \& HUTCHINSON, I.C. 1980. Effects of smelter pollutant on forest leaf litter decomposition near a nickel-copper smelter at Sudbury, Ontario. Canadian Journal of Botany 58:1722-1736.

GADD, G.M. 1992. Metals and microrganisms: a problem of definition. FEMS Microbiological Letters 100:197-204.

GADD, G.M. 1993. Interactions of fungi with toxic metals. New Phytologist 124:25-60.

GADD, G.M., RAMSAY, L., CRAWFORD, J.W. \& RITZ, K. 2001. Nutritional influence on fungal colony growth and biomass distribution in response to toxic metals. FEMS Microbiology Letters 204:311-316.

GAZARINI, L.C. 1983. Alguns aspectos ecológicos da macrófita Mayaca fluviatilis Aublet na Represa do Lobo (Brotas - Itirapina, SP). Dissertação de mestrado, Universidade Federal de São Carlos, São Carlos.

HENDRICKS, C.W. 1997. The effect of toxic chemicals on nutrient cycling processes in soils. In Soil ecotoxicology (J.Tarradellas, G. Bitton, \& D. Rossel, eds.), Lewis Publishers, New York, 235-270.

JORDAN, M.J. \& LECHEVALIER, P. 1975. Effects of zincsmelter emissions on forest soil microflora. Canadian Journal of Microbiology 21:1855-1865. 
KIRK, J.L., BEAUDETTE, L.A., HART, M., MOUTOGLIS, P., KLIROMOS, J.N., LEE, H. \& TREVORS, J.T. 2004. Methods of studying soil microbial diversity. Journal of Microbiological Methods 58:169-188.

KUSHNER, D.J. 1993. Effects of speciation of toxic metals on their biological activity. Water Pollution Research Journal of Canada 28:111-128.

NORDGREN, A., BÅ̊̊TH, E. \& SÖDERSTROM, B. 1983. Microfungi and microbial activity along a heavy metal gradient. Applied and Environmental Microbiology 45:1829-1837.

NORDGREN, A., BÅ̊̊TH, E. \& SÖDERSTROM, B. 1985. Soil microfungi in an area polluted by heavy metals. Canadian Journal of Botany 63:448-455.

PAUL, E.A. \& CLARK, F. E. 1996. Soil microbiology and biochemistry $2^{\text {nd }}$ ed., Academic Press, San Diego.

PRIMAVESI, A. 1990. Manejo ecológico do solo: a agricultura

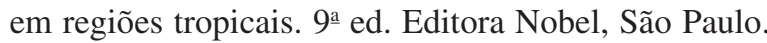

RAJAPAKSHA, R.M.C.P., TOBOR-KAPON, M.A. \& BÄ̈̈TH, E. 2004. Metal toxicity affects fungal and bacterial activities in soil differently. Applied and Environmental Microbiology 70:2966-2973.

ROSS, I.S. 1975. Some effects of heavy metals on fungal cells. Transactions of the British Mycological Society 64:75-193.

ROSS, I.S. 1993. Membrane transport processes and response to exposure to heavy metals. In Stress tolerance of fungi (D.H. Jennings ed.). Marcel Dekker, New York, p. 97-125.

SANTIAGO, A.L.C.M. \& SOUZA-MOTTA, C.M. 2006. Mucorales isolados do solo de mineração de cobre e produção de amilase e inulinase. Acta Botanica Brasílica 20:641-647.

SANTOS, V.B., WELLBAUM, C. \& SCHOENLEINCRUSIUS, I.H. 1998. Fungos filamentosos do solo da Ilha dos Eucaliptos na Represa do Guarapiranga em São Paulo, SP. Revista Brasileira de Botânica 12: 101-110.
SCHOENLEIN-CRUSIUS, I.H., PIRES-ZOTTARELLI, C. L.A. \& OKINO, L.K. 1994. Primeiro catálogo da coleção de culturas de fungos do Instituto de Botânica / Secretaria de Estado do Meio Ambiente, Instituto de Botânica, São Paulo.

SCHOENLEIN-CRUSIUS, I.H., MILANEZ, A.I., TRUFEM, S.F.B., PIRES-ZOTTARELLI, C.L.A., GRANDI, R.A.P., SANTOS, M.L. \& GIUSTRA, K.C. 2006. Microscopic fungi in the atlantic rainforest in Cubatão, SP, Brazil. Brazilian Journal of Microbiology 37:244-252.

TATSUYAMA, K., EGAWA, H., YAMAMOTO, H. \& INOUE, K. 1974. Influence of metallic compounds on soil microflora in farm polluted by chemicals. Transactions of the Mycological Society of Japan 15:18-22.

TATSUYAMA, K., EGAWA, H., SENMARU, H., YAMAMOTO, H., ISHIOKA, S., TAMATSUKURI, T. \& SAITO, K. 1975. Paecilomyces lilacinus: its tolerance to cadmium. Experientia 31:1037-1038.

TATSUYAMA, K., EGAWA, H., SENMARU, H., YAMAMOTO, H., ISHIOKA, S., TAMATSUKURI, T. \& SAITO, K. 1977. Cadmium and copper distributions in water and soil of Mt. Homanzan and its neighbourhood. Bulletin of the Faculty of Agriculture. Shimane University 11:18-22.

TREVORS, J.T. 1998. Bacterial biodiversity in soil with an emphasis on chemically contamined soils. Water, Air and Soil Pollution 101:45-67.

WAINWRIGHT, M. \& GADD, G.M. 1997. Fungi and industrial pollutants. In The Mycota. Environmental and microbial relationships, IV. (D.T.Wicklow \& B. Söderström, eds.) Springer Verlag, Berlin, p. 85-97.

WARCUP, J.H. 1950. The soil plate method for isolations of fungi from soil. Nature 166:117-118.

WELLBAUM, C., SCHOENLEIN-CRUSIUS, I.H. \& SANTOS, V.B. 1999. Fungos filamentosos em folhas do ambiente terrestre e aquático da Ilha dos Eucaliptos, Represa do Guarapiranga, São Paulo, SP. Revista Brasileira de Botânica 22:1-12. 